\title{
Lamivudine, a Reverse Transcriptase Inhibitor, Rescues Cognitive Deficits in a Mouse Model of Down Syndrome
}

Maria Martinez de Lagran

Centre for Genomic Regulation

Aleix Elizalde-Torrent

IrsiCaixa AIDS Research Institute, Badalona, Spain

Roger Paredes

IrsiCaixa AIDS Research Institute, Badalona, Spain

Bonaventura Clotet

IrsiCaixa AIDS Research Institute, Badalona, Spain

Mara Dierssen ( $\nabla$ mara.dierssen@crg.eu )

Centre for Genomic Regulation

\section{Research Article}

Keywords: LAMIVUDINE, REVERSE TRANSCRIPTASE INHIBITOR, RESCUES COGNITIVE DEFICITS, MOUSE MODEL

Posted Date: December 20th, 2021

DOI: https://doi.org/10.21203/rs.3.rs-1158032/v1

License: (c) (i) This work is licensed under a Creative Commons Attribution 4.0 International License. Read Full License 


\section{Abstract}

An elevated activity of retrotransposons is increasingly recognized to be implicated in a wide range of neurodegenerative and neurodevelopmental diseases. Down syndrome (DS) is the most common genetic disorder associated with intellectual disability and a genetic form of Alzheimer's disease. For this reason, we hypothesized that treatment with reverse transcriptase inhibitors could ameliorate DS phenotypes. In this proof of concept study, we treated trisomic (Ts65Dn) mice, a model of DS, with lamivudine, a reverse transcriptase inhibitor. We detected a significant improvement of neurobehavioral phenotypes, and a complete rescue of the hippocampal-dependent recognition memory upon treatment with lamivudine. Despite clinical studies in patients with DS are warranted, this study lays the groundwork for a novel and actionable therapeutic approach.

\section{Introduction}

Down syndrome (DS) is the most common known genetic disorder associated with moderate to severe intellectual disability due to a total or partial trisomy of the autosomal chromosome 21 (HSA21) and a genetic form of Alzheimer's disease (AD)[1]. Overexpression of HSA21 gene products such as DYRK1A[2], SOD1[3] or S100B[4] has been proposed to contribute to the neurological and neurobehavioral DS phenotypes. However, treatments targeted to candidate genes[6] or mechanisms have only been partially successful[7]. This is possibly explained because besides the HSA21 gene-dosage effects, triplication of HSA21 also leads to a genome wide transcriptional deregulation[5].

Recently, single-nucleus long-read RNA sequencing has revealed thousands of unannotated RNA transcripts containing intra-exonic junctions, involving a myriad of genes, including the amyloid precursor protein (APP)[8]. Notably, increased brain transcription and increased copy numbers of $A P P$ have been linked to $A P P$ somatic gene recombination associated with sporadic $A D$ and with $D S$, and could contribute to their cognitive deficits[9]. Retrotransposable elements like the long interspersed nuclear element 1 (LINE1 or L1 - 6kb) are thought to participate in this process in mammals[10]. Increased retrotransposition is observed in cell senescence and with ageing[11] and has been implicated in several neurodegenerative diseases including $A D$, frontotemporal dementia, prion disease, but also in developmental disorders such as Rett's syndrome, autism or fragile X syndrome[12].

Nucleoside reverse transcriptase inhibitors (NRTIs) have been used to suppress retrotransposition, and thus NRTIs could theoretically improve these pathologies. Importantly, in patients with HIV-1 NRTIs treatment is associated with reduced risk of HIV-associated neurocognitive disorder, AD, Parkinson's disease, and other dementias[13]. Preclinical studies have shown that the reverse transcriptase inhibitor lamivudine [(-)-L-2',3'-dideoxy-3'-thiacytidine (3TC)] improves cognition in senescence-accelerated prone 8 (SAMP8) mice, a model for studying human ageing and age-related diseases[14] and treatment with NRTIs doubled the lifespan of progeroid Sirt6-deficient mice[15], supporting this possibility. Last year a clinical trial to investigate the safety and feasibility of antiretroviral therapy with lamivudine for Alzheimer's disease was launched [ClinicalTrials.gov Identifier: NCT04552795]. Although a contribution of 
retrotransposition to developmental disorders has also been suggested and despite the close relationship of DS with AD[16], NRTIs have not been explored in DS. In this proof of concept study, we show a clear rescuing effect of lamivudine on hippocampal-dependent recognition memory in Ts65Dn mice, a DS mouse model.

\section{Material And Methods}

Trisomic (Ts65Dn, TS) male mice were obtained by breeding B6EiC4Sn.BLiA-Ts(1716)65Dn/DnJ females with C57BL/ $6 \times 6 \mathrm{JOlaHsd}$ (B6C3F1/OlaHsd) hybrid males. Mice were genotyped, and $\sim 25 \%$ of the offspring presented with trisomy. Wild-type (WT) littermates were used as controls. After weaning male mice were group housed with 3-4 animals per cage on a conventional 12:12 light cycle in controlled environmental conditions of humidity $(50-70 \%)$ and temperature $\left(21 \pm 1^{\circ} \mathrm{C}\right)$. Water and standard rodent chow were available ad lib. Experiments were conducted using 3-4 month-old male mice. TS and WT mice were assigned using a simple randomization to either control conditions or lamivudine $3 \mathrm{mg} / \mathrm{kg}$ (Epivir, 10 $\mathrm{mg} / \mathrm{ml}$, oral solution) in the drinking water. 15 TS and 20 WT mice were treated with lamivudine and 14 TS and 18 WT mice were used as a control group and received water. The treatment lasted 4 months. Behavioral testing was performed before the treatment and 1 and 4 months after starting the treatment. Behavioral experiments were conducted during the light phase of the light/dark cycle between $10 \mathrm{am}$ and $2 \mathrm{pm}$, and performed by trained observers blind to genotype. The novel object recognition test and plus maze were videotaped using a computer-assisted data acquisition system (SMART, Panlab Harvard Apparatus, Spain). Less stressful tests were performed first and each behavioral test was separated by at least $24 \mathrm{~h}$. The order of the tests was as follows: locomotor activity, novel object recognition, and elevated plus maze. All procedures had authorization from the Barcelona Biomedical Research Park Animal Research Ethics Committee (PRBB-CEEA) and the local government (Generalitat de Catalunya) and were conducted according to the European Directive 2010/63/EU and Spanish regulations RD 53/2013.

\section{Locomotor activity}

Spontaneous locomotor activity in the home cage was measured using an automated infrared light-beam monitoring system (ActiMot2, TSE system, Germany) and the ambulatory beam breaks (X axis) were counted for $24 \mathrm{~h}$. Total general activity was analyzed. Mice were isolated for assessing locomotor activity during 24 hours and immediately regrouped after the test.

\section{Novel object recognition test}

The apparatus consisted of a rectangular open-field arena (50 cm long $\times 50 \mathrm{~cm}$ wide $\times 50 \mathrm{~cm}$ high) made of black Plexiglas. Animals' behavior was monitored using System Motor Activity Record and Tracking software (SMART, Panlab Harvard Apparatus, Spain). On the first day, mice were habituated to the arena for $5 \mathrm{~min}$. On the second day, mice were presented with two identical objects, for $10 \mathrm{~min}$. Subjects failing to complete a minimum of $20 \mathrm{~s}$ of exploration during the familiarization session were excluded for 
posterior analysis. $24 \mathrm{~h}$ after (test session), mice were presented with one familiar object and one novel one, for $5 \mathrm{~min}$. The discrimination index was calculated as ((time exploring the novel object - time exploring the familiar object)/total time of exploration) * 100 [48]. Exploratory behavior was defined as the animal directing its nose towards the object at a distance of $<2 \mathrm{~cm}$ and manually registered by the experimenter. Sitting on or resting against the object was not considered as exploration. All the objects used were plastic made and induced similar exploration levels. The arena and objects were deeply cleaned between animals to avoid olfactory cues.

\section{Elevated plus maze}

The elevated plus maze paradigm was used to study anxiety-related behavior. The apparatus consists of a cross-shaped platform (four arms faced two to two with a length of $40 \mathrm{~cm}$ and $8 \mathrm{~cm}$ width) elevated 40 $\mathrm{cm}$ from the floor. Two opposing arms are protected by black walls and the other two are left unprotected. Because of the distance to the ground, the open arm is an aversive environment for the mouse, so it tends to remain in the closed arms. The proportion of time spent in open versus closed arms during $5 \mathrm{~min}$ is considered a measure of anxiety. Distance travelled was analyzed as a measure of the activity in the apparatus.

\section{Statistical analyses}

Two-way analysis of variance (ANOVA) repeated measures was used for testing genotype and treatment differences in the locomotor activity and elevated plus maze test, and for the analysis of the novel object recognition test. Tukey or Bonferroni post hoc tests were used as a correction between pairwise comparisons. All statistical analyses were performed using Statistical Package for the Social Sciences (SPSS) software (version 19.0) and GraphPad Prism (version 8.01).

\section{Results}

\section{Lamivudine ameliorated hyperactivity in TS mice}

We analyzed locomotor activity of WT and TS mice in baseline conditions and the effect of lamivudine in both genotypes one and four months after starting the treatment. Two-way ANOVA repeated measures showed a significant genotype effect $(F(1,22)=15.76 p<0.01)$. In baseline conditions, TS mice showed significantly increased total locomotor activity as compared to WT mice (Bonferroni post-hoc, $p<0.005$, Figure $1 \mathrm{~A}$ ), as shown by an increased distance travelled during 24 hours.

After one month of treatment with lamivudine, total locomotor activity was reduced in TS mice with respect to baseline levels, although not completely rescued to WT levels (Bonferroni post-hoc, $p<0.05$, Figure $1 \mathrm{~A}$ ), and the same tendency was observed after four months of treatment, although no statistically significant differences were observed between both genotypes (Bonferroni post-hoc, N.S., Figure 
1A). Two-way repeated measures ANOVA revealed a significant effect of lamivudine (treatment effect (F2, $60)=8.07, p<0.005)$. However, the distance travelled was significantly reduced after one month of treatment only in TS mice (Tukey post-hoc, TS basal vs TS-1M; $\mathrm{p}<0.05$ ).

These results indicate an amelioration of hyperactivity in Ts65Dn after treatment with lamivudine. When analyzing the circadian pattern of activity (Figure 1B), we observed that the treatment-associated changes of locomotor activity are mainly detected during the active (dark) phase of the circadian cycle. The increase in locomotor activity in TS mice during the dark phase are significant in basal conditions (One way ANOVA repeated measures, $F(1,33)=13.53, p<0.005$ ), and are significantly reduced with the treatment ( 1 month of treatment: $F(1,33)=9.09, p<0.01$ and 4 month of treatment $F(1,33)=5.88, p<$ 0.05).

\section{Lamivudine increased anxiety-like behavior in WT and TS mice}

We also assessed anxiety-like behavior in the elevated plus maze paradigm to understand whether TS mice would be more sensitive to this side effect of lamivudine (Figure 1C-E). We first analyzed locomotor activity (distance travelled) (Figure 1C). We did not detect genotype-dependent differences, but both genotypes travelled a reduced distance in the maze after treatment (Two-way ANOVA repeated measures, treatment effect $F(2,51)=34.67, \mathrm{p}<0.005$. Tukey's post-hoc test, WT BASAL vs WT1M $\mathrm{p}<0.005$, WTBASAL vs WT4M $\mathrm{p}<0.005$, TSBASAL vs TS1M $\mathrm{p}<0.05$, TS BASAL vs TS4M $\mathrm{p}<0.005$, Figure 1C). Possibly related to this, although no significant differences between genotypes were detected in the number of entries in open or closed arms, upon treatment we detected a reduction of entries in both open (Two-way ANOVA repeated measures, treatment effect, $F(1,38)=15.10, p<0.005$, Figure 1D) and closed arms (Two-way ANOVA repeated measures, treatment effect, $F(2,52)=12.55, p<0.005$ ). The reduction in the number of entries was maintained along the treatment at 1 and 4 months both in open (Tukey's posthoc test, WT-BASAL vs WT-1M p<0.005; WT-BASAL vs WT-4M $\mathrm{p}<0.005$; TS-1M $v s$ TS-4M p<0.05) and in closed arms (Tukey's post-hoc test, WT-BASAL vs WT-1M p<0.01; WT-BASAL vs WT-4M p < 0.05; TSBASAL vs TS-4M p<0.05, TS-1M vs TS-4M p<0.05). As both the entries in open and closed arm were reduced, this global reduction in activity as measured also by the distance travelled may reflect the habituation to the apparatus after repeated exposures.

Anxiety-like behavior is expressed as an increased time spent in the close arms with respect to open arms. No differences between genotypes in the number of entries nor in the time spent in neither open nor closed were observed, indicating similar anxiety-related behavior in TS and WT mice. However, we detected a treatment effect in the time spent in closed arms (Two-way ANOVA repeated measures, treatment effect, $F(1.5,46)=15.18, p<0.005$, Figure 1E), that was slightly increased in both genotypes and in the center (Two-way ANOVA repeated measures, treatment effect, $F(1.8,55)=11.82, p<0.005$ ), in which permanence was slightly reduced. Upon lamivudine treatment, WT mice spent significantly more time in closed arms (Tukey's post-hoc test, WT-BASAL vs WT-1M p < 0.005; WT-BASAL vs WT-4M p < 
0.005) and less time in open arms (non-significant) and in the center of the maze (Tukey's post-hoc test, WT-BASAL vs WT-1M p < 0.005; WT-BASAL vs WT-4M p < 0.005) as compared to basal conditions, suggesting anxiety-like effects. In the case of the TS, although the tendency is similar, no significant differences were observed.

\section{Lamivudine rescues recognition memory deficits in TS mice}

Recognition memory was evaluated using the novel object recognition paradigm that has been shown to be a robust and reproducible test for studying the Ts65Dn strain[17]. For this test, we included an independent group of WT and TS receiving water in basal conditions and after 1 or 4 months to discard possible carry-over effects of the test, and track the possible age-related differences in recognition memory. During the familiarization test, no differences in the time of exploration were observed among the groups at baseline, 1 month or 4 months (Figure 2A). In basal conditions, TS mice showed recognition memory impairment, as shown by the significantly reduced discrimination index (Two-way ANOVA, $F(3,47)=1.28 p<0.005$, Tukey's post-hoc test: WT-NT vs TS-NT $\mathrm{p}<0.01$ WT-T $v s$ TS-T $p<0.05)$. One month after starting non-treated mice continued showing cognitive impairment (Two-way ANOVA, $F(3,47)=5.27 p<0.005$, Tukey's post-hoc test: WT-NT $v s$ TS-NT $p<0.05)$, while TS treated with lamivudine, showed a complete rescue of the cognitive deficit reaching WT performance levels (Tukey's post-hoc test: WT-T $v s$ TS-T $p=0.98$, Figure 2B). The cognitive rescue in TS mice was maintained after 4 months of treatment (Two-way ANOVA, F(3,47) $=6.28 p<0.005$, Tukey's post-hoc test: WT-T $v s$ TS-T $p=$ 0.82 , Figure $2 \mathrm{~B}$ ), whereas non-treated Ts65Dn mice showed an even worse discrimination index (Tukey's post-hoc test: WT-NT vs TS-NT $\mathrm{p}<0.005$, Figure $2 \mathrm{~B}$ ), probably due to age-associated cognitive decline.

\section{Discussion}

Increased retrotransposition is implicated in neurodevelopmental diseases, so in this proof of concept study we tested if lamivudine, a reverse transcriptase inhibitor, could reverse the cognitive impairment in a DS mouse model, the Ts65Dn (TS) mice, the most extensively validated model of DS. TS mice present hyperactivity and hippocampal dependent learning deficits[18], and recapitulate several of the DS neurobehavioral and cognitive phenotypes. Lamivudine significantly improved neurobehavioral phenotypes and completely rescued hippocampal-dependent recognition memory in young adult TS mice. It also led to a slight increase of anxiety-related behavior, though only in wild types.

We could speculate several mechanisms contributing to the neurobehavioral and cognitive improvement. It has been previously reported that upregulation of Line1 triggers an IFN-I response in Sirt6-knockout and normal aged mice, and that pathologies and lifespan of Sirt6 progeroid mice can be improved with NRTIs[15]. Recent work showed a constant activation of the type 1 interferon (IFN-I) response in DS[19] that may contribute to brain neuropathology, as the IFN-I response is characteristic of cellular senescence[20]. Moreover, in DS, inflammatory biomarkers are strongly associated with intellectual disability and obesity risk $[21,22]$. The fact that lamivudine rescued the cognitive phenotype suggests 
that IFN-I overactivation in DS[23] may be contributed by increased retrotransposition, driven by LINE-1 reactivation. De Cecco et al. [24] found that IFN-I response is overactive in human senescent cells and in aged mice, where treatment with lamivudine downregulated IFN-I activation and age-associated inflammation[24]. Individuals with trisomy 21 start to age prematurely[25] presenting early in life conditions such as $\mathrm{AD}$, strongly contributed by overexpression of APP or DYRK1A. Increased copy numbers and overexpression of $A P P$ gene associated with sporadic AD have been linked to APP somatic gene recombination[26]. This form of gene recombination produces internally truncated RNA sequences containing intra-exonic junctions (IEJs), and a recent study using single nucleus RNA sequencing [8] has shown novel truncated RNAs containing IEJs and involving not only APP but thousands of other genes in DS. It might thus be speculated that the observed beneficial effects of lamivudine could be due to the inhibition of the generation of one or more non-classical variant(s) of the APP gene, as APP overexpression is involved in DS phenotypes[27].

In summary, our study demonstrated for the first time that lamivudine improves cognition in a mouse model of DS, providing experimental evidence for the use of reverse transcriptase inhibitors as a new potential treatment for ameliorating cognitive impairment in DS. Further studies to assess safety and tolerability in $\mathrm{DS}$ patients with early stage $A D$ are warranted.

\section{Declarations}

\section{Author Contributions:}

$\mathrm{MD}, \mathrm{BC}$ and RP conceived the paper, MMdL performed and analyzed the experiments. MD, MMdL and AET wrote the manuscript. All the authors revised and approved the last version of the manuscript.

\section{Funding:}

The lab of M.D. is supported by the Departament d'Universitats, Recerca i Societat de la Informació de la Generalitat de Catalunya (Grups consolidats 2017 SGR 926, 2017 SGR 138). This research was funded by the Agencia Estatal de Investigación (PID2019-110755RB-I00/AEI/10.13039/501100011033), the European Union's Horizon 2020 research and innovation programme under grant agreement No 848077. This reflects only the author's view and the European Commission is not responsible for any use that may be made of the information it contains. Jerôme Lejeune Foundation (Grant number 2002), NIH (Grant Number: 1R01EB 028159-01), Marató TV3 (\#2016/20-30), and JPND (Heroes AC170006). The CRG acknowledges the support of the Spanish Ministry of Science and Innovation to the EMBL partnership, the Centro de Excelencia Severo Ochoa, and the CERCA Programme/Generalitat de Catalunya. The CIBER of Rare Diseases (CIBERER) is an initiative of the ISCIII.

\section{Institutional Review Board Statement:}


The study was conducted according to the guidelines of the local (law 32/2007) and European regulations (2010/63/EU) and the Standards for Use of Laboratory Animals no. A5388-01 (NIH), and approved by the Ethics Committee of Parc de Recerca Biomèdica (Comité Ético de Experimentación Animal del PRBB (CEEA-PRBB); MDS 18-0031). The CRG is authorized to work with genetically modified organisms (A/ES/05/I-13 and A/ES/05/14).

\section{Data availability:}

The original contributions presented in the study are included in the article/Supplementary Material, further inquiries can be directed to the corresponding author.

\section{Acknowledgments:}

We thank Cèsar Sierra for critical reading of the manuscript.

\section{Conflicts of Interest:}

The authors declare no conflict of interest

\section{References}

1. Dierssen, M., Down syndrome: the brain in trisomic mode. Nat Rev Neurosci, 2012. 13(12): p. 844-58.

2. Altafaj, X., et al., Neurodevelopmental delay, motor abnormalities and cognitive deficits in transgenic mice overexpressing Dyrk1A (minibrain), a murine model of Down's syndrome. Hum Mol Genet, 2001. 10(18): p. 1915-23.

3. Gulesserian, T., et al., Superoxide dismutase SOD1, encoded on chromosome 21, but not SOD2 is overexpressed in brains of patients with Down syndrome. J Investig Med, 2001. 49(1): p. 41-6.

4. Griffin, W.S., et al., Brain interleukin 1 and S-100 immunoreactivity are elevated in Down syndrome and Alzheimer disease. Proc Natl Acad Sci U S A, 1989. 86(19): p. 7611-5.

5. De Toma, I., C. Sierra, and M. Dierssen, Meta-analysis of transcriptomic data reveals clusters of consistently deregulated gene and disease ontologies in Down syndrome. PLoS Comput Biol, 2021. 17(9): p. e1009317.

6. de la Torre, R., et al., Safety and efficacy of cognitive training plus epigallocatechin-3-gallate in young adults with Down's syndrome (TESDAD): a double-blind, randomised, placebo-controlled, phase 2 trial. Lancet Neurol, 2016. 15(8): p. 801-810.

7. Fernandez, F. and J.O. Edgin, Pharmacotherapy in Down's syndrome: which way forward? Lancet Neurol, 2016. 15(8): p. 776-777. 
8. Palmer, C.R., et al., Altered cell and RNA isoform diversity in aging Down syndrome brains. Proc Natl Acad Sci U S A, 2021. 118(47).

9. Kaeser, G.E. and J. Chun, Mosaic Somatic Gene Recombination as a Potentially Unifying Hypothesis for Alzheimer's Disease. Front Genet, 2020. 11: p. 390.

10. Muotri, A.R., et al., Somatic mosaicism in neuronal precursor cells mediated by $L 1$ retrotransposition. Nature, 2005. 435(7044): p. 903-10.

11. Gorbunova, V., et al., The role of retrotransposable elements in ageing and age-associated diseases. Nature, 2021. 596(7870): p. 43-53.

12. Li, W., et al., Transposable elements in TDP-43-mediated neurodegenerative disorders. PLoS One, 2012. 7(9): p. e44099.

13. Siangphoe, U., et al., Associations of antiretroviral therapy and comorbidities with neurocognitive outcomes in HIV-1-infected patients. AIDS, 2020. 34(6): p. 893-902.

14. Li, M., et al., Lamivudine improves cognitive decline in SAMP8 mice: Integrating in vivo pharmacological evaluation and network pharmacology. J Cell Mol Med, 2021. 25(17): p. 84908503.

15. Simon, M., et al., LINE1 Derepression in Aged Wild-Type and SIRT6-Deficient Mice Drives Inflammation. Cell Metab, 2019. 29(4): p. 871-885 e5.

16. Gardner, E.J., et al., Contribution of retrotransposition to developmental disorders. Nat Commun, 2019. 10(1): p. 4630.

17. Sierra, C., et al., Social Factors Influence Behavior in the Novel Object Recognition Task in a Mouse Model of Down Syndrome. Front Behav Neurosci, 2021. 15: p. 772734.

18. Escorihuela, R.M., et al., Impaired short- and long-term memory in Ts65Dn mice, a model for Down syndrome. Neurosci Lett, 1998. 247(2-3): p. 171-4.

19. Sullivan, K.D., et al., Trisomy 21 consistently activates the interferon response. Elife, 2016. 5.

20. Gensous, N., et al., Down syndrome, accelerated aging and immunosenescence. Semin Immunopathol, 2020. 42(5): p. 635-645.

21. Manti, S., et al., Inflammatory biomarkers and intellectual disability in patients with Down syndrome. J Intellect Disabil Res, 2018. 62(5): p. 382-390.

22. Fructuoso, M., et al., Increased levels of inflammatory plasma markers and obesity risk in a mouse model of Down syndrome. Free Radic Biol Med, 2018. 114: p. 122-130.

23. Ling, K.H., et al., Functional transcriptome analysis of the postnatal brain of the Ts1Cje mouse model for Down syndrome reveals global disruption of interferon-related molecular networks. BMC Genomics, 2014. 15: p. 624.

24. De Cecco, M., et al., L1 drives IFN in senescent cells and promotes age-associated inflammation. Nature, 2019. 566(7742): p. 73-78.

25. Patterson, D. and D.C. Cabelof, Down syndrome as a model of DNA polymerase beta haploinsufficiency and accelerated aging. Mech Ageing Dev, 2012. 133(4): p. 133-7. 
26. Lee, M.H., et al., Somatic APP gene recombination in Alzheimer's disease and normal neurons. Nature, 2018. 563(7733): p. 639-645.

27. Rodgers, S.P., et al., Transgenic APP expression during postnatal development causes persistent locomotor hyperactivity in the adult. Mol Neurodegener, 2012. 7: p. 28.

\section{Figures}

A

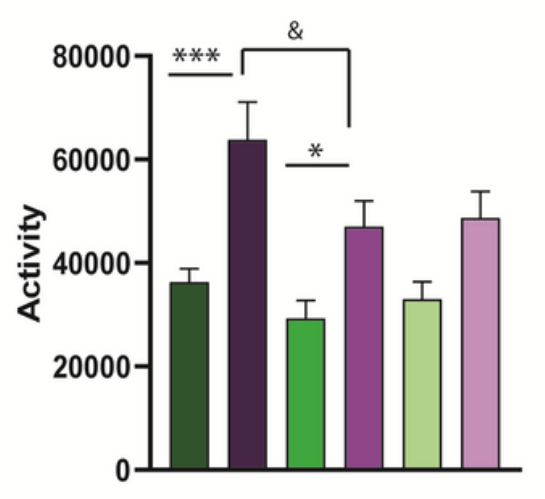

C

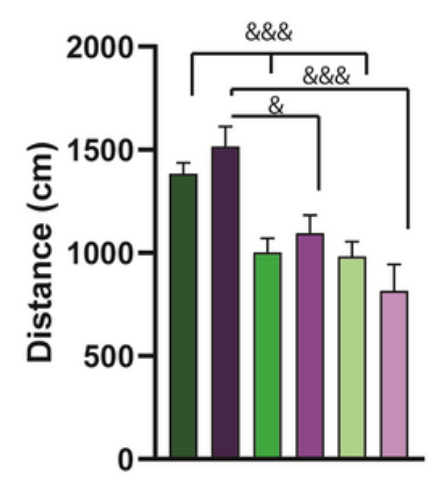

D
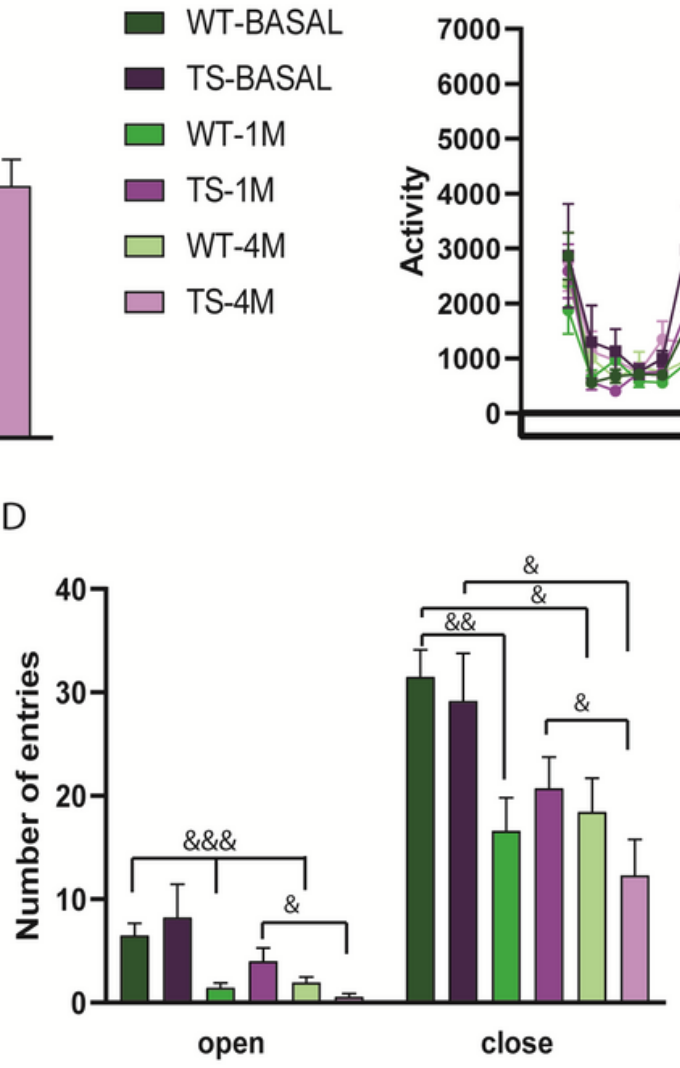

B

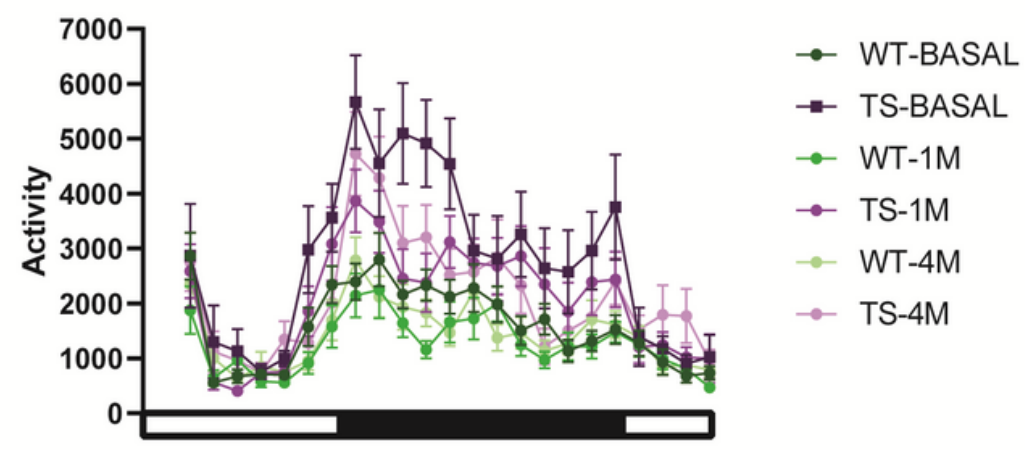

$E$

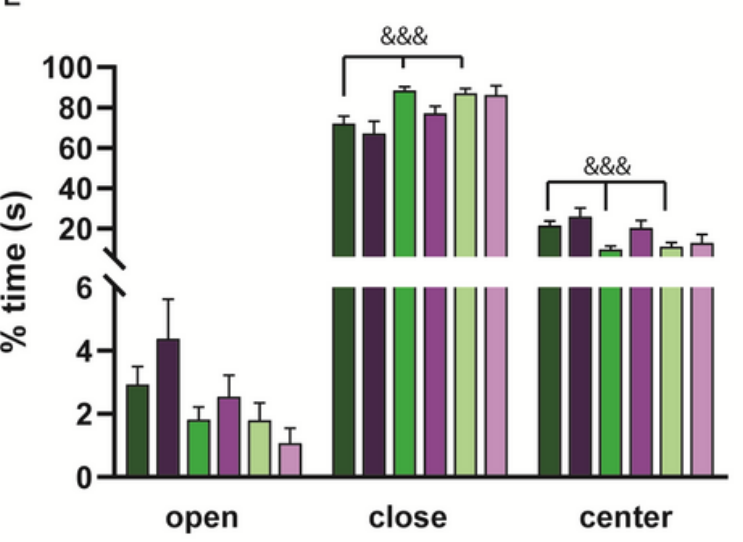

Figure 1

Locomotor activity and anxiety-like behavior in TS and respective WT in basal conditions and after 1 or 4 months of treatment with lamivudine. The upper panel depicts $\mathbf{A}$. total activity and $\mathbf{B}$. circadian activity for 24 hours measured as ambulatory (X axis) beam breaks. In B. the dark square under the X-axis represents the dark phase of the cycle (WT $n=20, T S n=15)$. Anxiety-like behavior was assessed in elevated plus maze. The lower panel depicts the $\mathbf{C}$. total distance travelled, D. number of entries and $\mathbf{E}$. percentage of time spent in each zone of the elevated plus maze (WT $n=20, T S n=12$ ). Data are represented as mean \pm SEM. Genotype effect * $p<0.05$, $* * p<0.01$, $* \star \star p<0.005$, Treatment effect \& $p$ $<0.05, \& \& p<0.01 ; \& \& \& p<0.005$. 
A

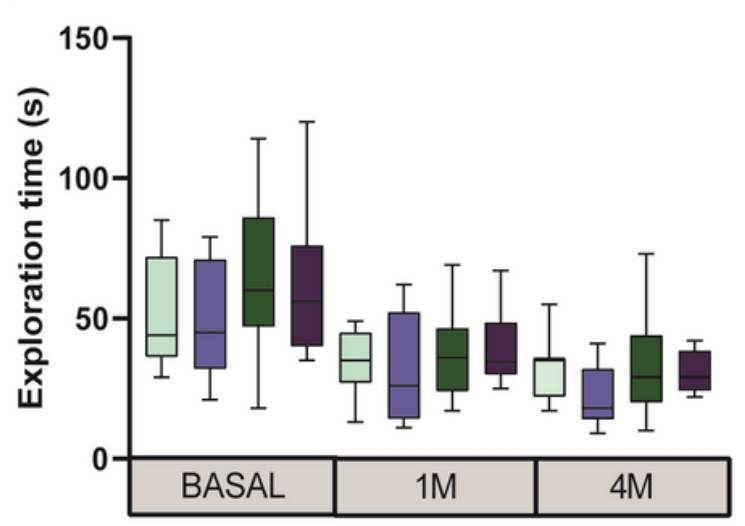

B

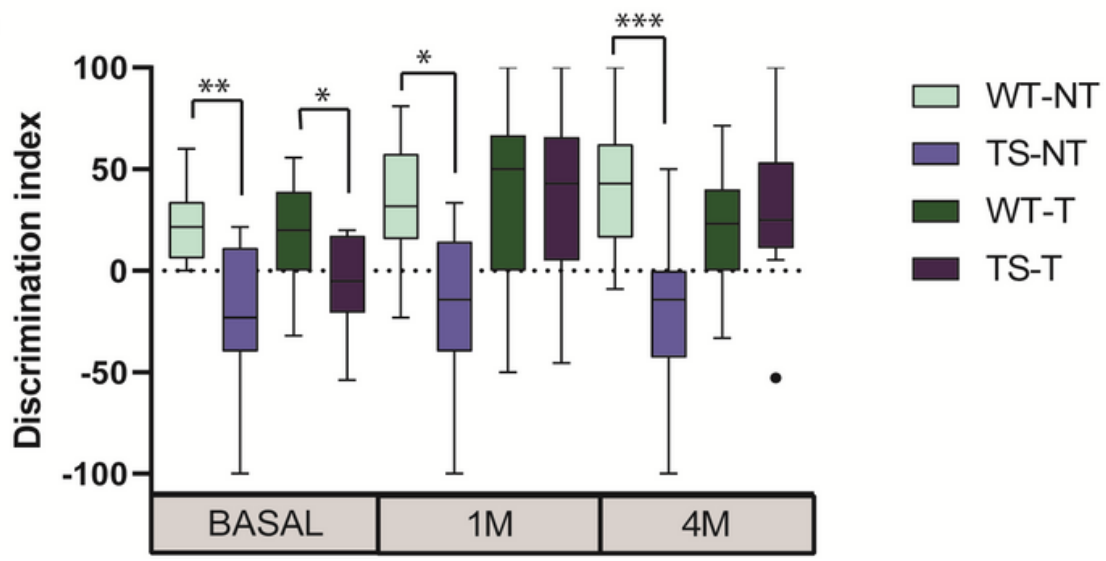

Figure 2

Cognitive assessment in novel object recognition test in TS and respective WT in basal conditions and after 1 or $\mathbf{4}$ months of treatment with lamivudine. Boxplots represent $\mathbf{A}$. total time of exploration during the familiarization phase and $\mathbf{B}$. the discrimination index 24 hours after familiarization (treated: Wild type (WT-T) $n=17$, Ts65Dn (TS-T) $n=13$; non-treated: Wild type (WT-NT) $n=9$, Ts65Dn (TS-NT) n = 9).

Boxplots extend from the $25^{\text {th }}$ to $75^{\text {th }}$ percentiles and the median is represented as a line in the box. The whiskers correspond to the maximum and minimum value excluding outliers. ${ }^{*} p<0.05,{ }^{\star \star} p<0.01$, ${ }^{\star \star \star} p$ $<0.005$.

\section{Supplementary Files}

This is a list of supplementary files associated with this preprint. Click to download.

- Supplementarymaterial.xlsx 